\title{
ILCEA
}

Revue de l'Institut des langues et cultures

d'Europe, Amérique, Afrique, Asie et Australie

$24 \mid 2015$

Lire et écrire ensemble

Une vie de Pierre Ménard de Michel Lafon : «Ces vies imaginaires, nostalgiques et méditerranéennes»

"Those Imaginary, Nostalgic, Mediterranean Lives" : Michel Lafon's Une vie de Pierre Ménard

\section{Carla Fernandes}

\section{(2) OpenEdition}

Journals

Édition électronique

URL : http://journals.openedition.org/ilcea/3643

DOI : 10.4000/ilcea.3643

ISSN : 2101-0609

Éditeur

UGA Éditions/Université Grenoble Alpes

Édition imprimée

ISBN : 978-2-84310-313-1

ISSN : 1639-6073

Référence électronique

Carla Fernandes, "Une vie de Pierre Ménard de Michel Lafon : "Ces vies imaginaires, nostalgiques et méditerranéennes » », ILCEA [En ligne], 24 | 2015, mis en ligne le 22 octobre 2015, consulté le 20 avril 2019. URL : http://journals.openedition.org/ilcea/3643 ; DOI : 10.4000/ilcea.3643

Ce document a été généré automatiquement le 20 avril 2019

(c) ILCEA 


\section{Une vie de Pierre Ménard de Michel Lafon : «Ces vies imaginaires, nostalgiques et méditerranéennes »}

"Those Imaginary, Nostalgic, Mediterranean Lives" : Michel Lafon's Une vie de Pierre Ménard

Carla Fernandes

1 Paru aux éditions Gallimard en 2008, portant sous le titre la mention « roman », Une vie de Pierre Ménard reçoit le Prix Valéry Larbaud 2009 et est publié en Argentine dans une traduction de César Aira en 2010 (éditions Lumen).

2 Je partirai ici de "Ces vies imaginaires, nostalgiques et méditerranéennes" tracées au stylo marron, couleur du tronc des arbres, par Michel dans l'exemplaire qu'il me faisait parvenir par courrier à l'été 2013. Ces six mots ont accompagné la première lecture d'Une vie de Pierre Ménard puis toutes les relectures. Au fil de leur progression le mot roman s'est dissout au profit d'une qualification générique polymorphe mais toujours attachée à l'idée de fragment: de prose, de vers, de fiction au sens borgésien. Chaque fragment semble avoir été tissé, puis serti pour lui-même, avant d'intégrer la trame générale, qui redonne vie au personnage de la fiction de Borges. Celui dont l'œuvre visible est énumérée au début du conte de Borges et dont l'œuvre invisible « consta de los capítulos IX y xxxviII de la primera parte de Don Quijote y de un fragmento del capítulo XXII.» (Borges, 2013: 45) Cuvre réécrite selon la logique du même et de l'identique, selon ce fragment anthologique :

No quería componer otro Quijote - lo cual es fácil- sino el Quijote. Inútil agregar que no encaró nunca una transcripción mecánica del original; no se proponía copiarlo. Su admirable ambición era producir unas páginas que coincidieran -palabra por palabra y línea por línea-con las de Miguel de Cervantes. (Borges, 2013 : 46)

Cette citation, en français, précède l'Avant-propos de l'œuvre. Comme n'a pas manqué de le faire remarquer Michel Lafon lui-même, l'entreprise littéraire de Pierre Ménard, telle que la présente la fiction de Borges, importe moins que l'une des vies possibles de son personnage. Néanmoins, précise-t-il dans un entretien: «El punto de partida no fue 
Borges ni Ménard sino mi fascinación por el jardín botánico de mi ciudad natal, Montpellier, una ciudad que no tenía literatura ni vida intelectual. Es una especie de revancha. En la novela la transformo en el centro secreto de la modernidad literaria. » (Lafon, 2009)

CEuvre-jardin, Une vie de Pierre Ménard ancre son espace littéraire dans ce territoire réel et imaginaire qu'est le Jardin des plantes de Montpellier, le plus ancien de France. Le Ménard nîmois de Jorge Luis Borges devient ainsi un personnage montpelliérain, qui parcourt et hante ce jardin, à l'origine universitaire. Centre d'un cosmos vital et littéraire, le jardin marque aussi la prédominance du règne végétal dans l'univers de la ville. Résumé de la littérature, le Jardin des Plantes est un des inédits de Ménard. Résumé du monde, le Jardin à l'origine abritait des plantes médicinales, susceptibles de faire avancer la science et d'allonger le temps de vie des hommes:

Depuis la fin du xvI siècle, le Jardin des plantes de Montpellier est universitaire. Comme la majorité des jardins botaniques créés à cette époque, il a été initialement conçu comme outil d'enseignement et de recherche dans le domaine de la santé humaine, à une époque où l'essentiel des médicaments étaient à base de préparations végétales. En 1593, Henri IV établit par lettre patente une cinquième régence (chaire) d'anatomie et de botanique à la Faculté de médecine de Montpellier. Dans une seconde lettre patente, il missionne le titulaire de cette chaire, Pierre Richer de Belleval, pour la création d'un jardin de démonstration des simples (plantes médicinales). (Michaud, 2011)

Michel Lafon consacre de nombreuses pages et plusieurs fragments clés au Jardin des plantes, territoire historique et réel mais aussi hors de l'espace et du temps : on y croise des « absents» (p. 22) ou encore, on le pratique comme le pratique Ménard « comme une consolation » (p. 31). Dans Une vie de Pierre Ménard le Jardin des plantes est un des inédits de Pierre Ménard, comme souligné précédemment, qui renvoie à la mise en récit de la réalité et au jeu de mise en abyme de la représentation. Compte tenu de ces différents niveaux des figures du récit, le Jardin est aussi un chronotope en tant que « corrélation essentielle des rapports spatiotemporels, telle qu'elle a été assimilée par la littérature » (Baktine, 1978 : 236-398). Le Jardin, espace qui irradie tous les espaces et les temps du roman, a partie liée avec l'amitié et la mort. Mort et amitié sont transposées de la fiction de Borges, « Pierre Ménard, autor del Quijote ».

Ainsi, après avoir passé en revue l'« œuvre visible» de Ménard, Maurice Legrand s'interroge :

Mais si j'imagine Ménard encore vivant en 1939, puis-je envisager Borges publiant quand même, cette année-là, sa "nécrologie »? Aurait-il poussé le tour de force jusqu'à commencer l'élégie fictive d'un ami vivant? Et y aurait-il mis, alors, plus de malice, plus de mordant, sans la pudeur nostalgique à laquelle oblige, malgré tout, l'inexorable? Qu'est-ce qui primait pour lui, en fin de compte, en ce début d'année 1939 : écrire sur Ménard, écrire sur un proche récemment disparu, écrire sur Cervantès? Méditer sur l'amitié, sur la mort, sur la littérature ? Ecrivant sur Ménard, il fit ces trois choses à la fois - c'est la seule certitude. (Lafon, 2008 : 37)

7 Issu d'une fiction oubliée, selon le romancier, le Ménard d'Une vie... fait nostalgiquement résonner amitié, mort et littérature à travers ses pages. 


\section{Construction et dessin général du roman-jardin}

Une vie de Pierre Ménard se compose d'un Avant-propos signé de Maurice Legrand, situé à Montpellier et daté du 24 août 1957. Il est suivi d'un tout aussi fictionnel Avertissement, attribué à l'éditeur et daté du 24 décembre 2008, année où paraît l'œuvre. Les seuils de celle-ci se complètent par un Post-scriptum dont la première ligne est significativement mise en exergue comme si elle constituait à elle seule l'unité d'un mince paragraphe : «Tout, dans une fiction, est fictif. »

Trois étapes essentielles de l'Avant-propos contribuent à dévoiler une trame faite de fragments, qui vont se succéder jusqu'au Post-scriptum. L'écriture raffinée et exigeante ainsi que la juxtaposition des fragments fait tôt oublier que cette clé de construction vient d'être fournie. Tout d'abord, pour se démarquer du modèle borgésien, tout en s'inscrivant dans la traditionnelle fusion vie-littérature et réalité-fiction, le narrateur établit que dans " Pierre Ménard, autor del Quijote ", la nouvelle contribue "à la transformation de l'homme en personnage » (Lafon, $2008: 12$ ).

Puis, le fictif Maurice Legrand retourne la nouvelle borgésienne et accorde à la réalité la primauté sur la fiction et à l'homme, Ménard, la primauté sur le personnage : «Pierre Ménard n'est pas une simple construction d'encre et de papier, il a existé, oui, je l'affirme, je le crie aux incrédules (ou aux trop crédules) : Pierre Ménard a existé, et j'ai eu le privilège d'être de ses proches! ( (Lafon, $2008: 13-14)$. Vingt ans séparent la réalisation du « livre de 'témoignage' [...] sur l'ami et l'écrivain » de la mort de celui-ci.

11 Le troisième niveau de signification renvoie aux éléments épars et pluriels qui composent ce singulier « livre de témoignage » : «morceaux de sa vie [...], notes prises par moi au fil de nos rencontres et de nos causeries, extraits de correspondances, inédits [...] (Lafon, 2008 : 14-15). Parmi les inédits, les pages récupérées du Jardin des plantes de Montpellier sont à prendre en compte, de même que quelques pages en français attribuées à Borges et des extraits copiés d'un Mémoire emprunté à un auteur montpelliérain. N'en déplaise à Borges, parmi les inédits de Ménard figure le roman L'Ile de Bloy (Lafon, 2008 : 15-16).

L'Avertissement apporte de la densité temporelle au témoignage, tout en le décalant et en le rendant anachronique et déphasé : un demi-siècle sépare le temps de l'histoire, qui se veut témoignage de celui de son édition, ultime étape de la vie d'une œuvre ici évoquée immédiatement après, dans un rapport de contiguïté permis par l'objet livre. Tous ces fragments servent de preuves manifestes et conservent témoignage de l'existence de Ménard. En même temps, le topos cervantin et borgésien du manuscrit trouvé y est retravaillé : « En fin de compte, Legrand n'a manifestement pas réussi à publier ces pages, si tant est qu'il s'y soit essayé. Le hasard ou quelque autre nécessité a voulu qu'elles demeurent, avec d'autres, dans un carton miraculeusement conservé au grenier d'une maison languedocienne, d'où l'on entreprend aujourd'hui de les tirer. » (Lafon, 2008 : 17)

Le Post-scriptum de M. Lafon revient sur le mouvement perpétuel entre la fiction et la réalité, à l'origine de l'œuvre de Miguel de Cervantes, tout autant que de celle de Jorge Luis Borges. Tautologique, il débute par «Tout, dans une fiction, est fictif. Tout, ou presque» (Lafon, 2008: 181) puis se conclut par « Tout, dans une fiction, absolument tout, comme on le voit, est bel et bien fictif » après avoir retracé une archéologie, non pas des sources réelles de ce que la fiction expose mais des divers types de récit, du plus minimal au plus volumineux, qui composent le fragmentaire « livre de témoignage ». Se succèdent 
ainsi la citation d'une fiction érigée en " pièce la plus authentique de ce roman » (Lafon, 2008 : 181) et un second paragraphe qui est une longue énumération scandée par les substantifs des débuts de phrase : l'évocation, le fragment, la formule, la thèse annonçant « la fulgurante prédiction de Macedonio Fernández au jeune Jorge Luis Borges » puis L'Ile de Bloy (Lafon, $2008: 181$ ). Le troisième paragraphe est tout entier consacré au Jardin des plantes de Montpellier, à la fois, espace littéraire et espace symbolique, œuvre dans l'œuvre, métaphore obsédante, mythe personnel et noyau générateur d'Une vie de Pierre Ménard. L'avant dernier paragraphe évoque la non-authentique présence à Montpellier de Borges lors de son voyage vers l'Espagne pendant la Première Guerre Mondiale et souligne l'invention de l'imposition du personnage littéraire Ménard, fait homme le temps d'Une vie de Pierre Ménard, à la réalité et à Borges lui-même. Le dernier paragraphe accorde à l'oubli la puissance de l'analogie et de la capacité créatrice renouvelée :

Je me rends compte aujourd'hui seulement, en écrivant les dernières lignes de ce post-scriptum, que je me suis trouvé, par rapport à «Pierre Ménard, auteur du Quichotte », dans une situation étrangement similaire à celle de Ménard face au roman de Cervantes: c'est longtemps après l'avoir lu et oublié que le Nîmois entreprit, selon Borges, sa tâche immense et dérisoire; moi-même, c'est dans le souvenir un peu lointain et tout compte fait un peu flou de cette nouvelle, [...], c'est dans l'écho fidèle ou infidèle de quelques-unes de ses phrases, et dans l'amitié de ses personnages, dans leur intimité attendrie que j'ai écrit ce roman. Moins héroïque que Ménard [...] j'ai renoncé d'emblée à tenter de produire quelques pages coïncidant, mot pour mot et ligne à ligne, avec celle de Borges. (Lafon, 2008 : 183)

Outre cette dernière, la différence essentielle reste la transposition du personnage de Ménard dans un autre espace, celui de Montpellier, et plus particulièrement la relation spécifique qui le lie au Jardin des plantes de cette ville.

\section{Le Jardin : texture et symboles}

Dès l'Avant-propos attribué à Maurice Legrand (Montpellier, 24 août 1957), sont évoqués deux inédits de Pierre Ménard qui renvoient au Jardin :

Parmi les inédits, sa "théorie des trois jardins » et surtout quelques pages de ce Jardin des plantes de Montpellier qui l'accompagna toute sa vie et où il a lui-même rassemblé, tout à la fois, son amour pour le cœur solitaire et sauvage de la vieille cité languedocienne, les promenades littéraires qu'il y fit avec d'autres bien plus illustres que moi, sa connaissance minutieuse des arbres et des simples, son secret voisinage, enfin, avec Pierre Richer de Belleval, fondateur admirable du Jardin - dont il rêvait sans doute de composer la biographie et à qui tant de passions, tant d'affinités le liaient, par-delà les siècles. (Lafon, $2008: 15$ )

Cette référence, au seuil du roman et alors que le processus de fictionnalisation se met en route, permet d'insérer un récit ou deux dans cet autre récit (principal) qu'est Une vie de Pierre Ménard. Impossible pour la fiction de rivaliser avec le réel et, d'emblée, ils sont enchâssés dans une déréalisation qui brouille et invalide leurs frontières.

Un inventaire des lieux dans Une vie de Pierre Ménard confirme la prépondérance du Jardin des plantes dans le monde que le roman construit. Dès le topos d'ouverture, il semble renvoyer non seulement (et logiquement) à un espace mais aussi à un temps, propice à la nostalgie, dans lesquels Legrand inscrit la première apparition de Pierre Ménard dont la position économise une première description physique :

Le soir, à l'heure tiède où le jour semble vaciller, je descendais par les rues de la vieille ville jusqu'au Jardin des plantes. Ayant écrit cette phrase, je m'arrête et je 
revois le temps que j'évoque : Montpellier, juillet 1919. [...] L'enchevêtrement des maisons et des ruelles, la cathédrale Saint Pierre comme un chat préhistorique adossé à la faculté de médecine, quelques longueurs encore de chaussée escarpée, enfin le boulevard désert et, sur le trottoir d'en face, les grilles du Jardin. J'entrais, je choisissais l'allée du milieu, la plus aventureuse [...] C'est là, face au tombeau qu'abrite cette niche, me tournant le dos, que je vis Pierre Ménard pour la première fois. (Lafon, $2008: 19-20)$

Rapidement dans l'évocation témoignage de Legrand, le lieu est associé à des rencontres et des promenades littéraires, qui placent Ménard en position d'aîné et d'initiateur de deux grands auteurs de la littérature française: Paul Valéry et André Gide, dans un second temps :

[...] Parler de littérature dans ce jardin avec un inconnu me ravit [...] Il me parle de son « jeune ami Paul Valéry » avec ferveur. C'est lui qui a enseigné le Jardin - «et tant d'autres choses »- à Valéry, à peine bachelier, et depuis lors ce territoire est devenu le leur, pour d'immuables rendez-vous. [...] « Tous les jardins sont, d'une manière ou d'une autre, comme ce parc du Petit Trianon où l'on peut croiser, à ce qu'on dit, des fantômes ou, en tout cas, des absents. (Lafon, $2008: 22$ )

19 Le cercle littéraire et amical, situé dans le Jardin, va en s'élargissant au cours de paragraphes de notes télégraphiques où le sujet des phrases, Ménard, est implicite et effacé (tout comme il est de dos dans le Jardin lors de la première évocation qu'en fait Legrand) :

Évoque aussi, dans cette lettre, la rencontre de Valéry et de Gide à Montpellier, en 1890, une rencontre dans laquelle il me confie avoir joué «un petit rôle, parallèlement à Pierre Louÿs ». Copie à mon intention cette phrase nostalgique - quasi élégiaque - de Gide, telle une vignette du Jardin. (Lafon, $2008: 23$ )

Puis, un paragraphe isolé des autres par des astérisques rapporte un fragment de lettre qui témoigne de son rôle d'initiateur et bientôt d'ami :

Gide à Ménard, revenant sur sa rencontre avec Valéry (lettre de mars 1896) : « Nos premières entrevues de Montpellier, grâce à vous et comme à votre ombre bienveillante, ont un charme de souvenir que je ne retrouve à rien d'autre, un charme tout particulier, presque indépendant de nous deux tant y aidaient la saison, les avenues du Peyrou et les allées du Jardin des plantes, la tombe de Narcissa, votre enseignement et votre amitié si sensibles - si présents - dans ce paysage, et notre âge. » (Lafon, $2008: 23$ )

21 Dans la texture du récit, autour du Jardin vont se dégager plusieurs unités narratives. La suite ne fera que confirmer qu'en même temps, il va lier entre eux les fragments les plus épars et établir un principe d'organisation. Le Jardin rempli ainsi la fonction de principe dynamique du récit. Et s'il unifie le récit fragmentaire, il l'amplifie aussi comme en témoigne l'importante séquence consacrée à l'amitié de Ménard avec le paysan ardéchois, qui lui a sauvé la vie pendant la Guerre et au Coiron où il habite.

Cette séquence est intitulée « Rêves d'Ardèche » (Lafon, 2008 : 90), titre qui ne va pas sans rappeler la rêverie associée symboliquement au Jardin, qui transporte hors du monde. Par contraste, le fragment débute par un rappel de la Grande Guerre et des dangers encourus par Ménard, engagé volontaire dans un hôpital. Lors d'une attaque de l'armée allemande, un paysan ardéchois lui sauve la vie. Après quelques lignes où cette Grande Guerre est écrite, le reste du fragment va être consacré à la célébration de la vie, de l'amitié, de l'été, de l'inspiration et de la force qu'apporte la nature et de l'état de création qu'elle permet:

Pour célébrer cette amitié, Ménard se rendait deux semaines, tous les mois d'août, sur le plateau du Coiron, où ce paysan avait sa ferme. Deux semaines de conversation à la veillée avec la famille Vidal, qui redécouvrait la guerre de son 
héros en l'entendant rire avec son «visiteur de la ville». Et de marches interminables, matin et soir, [...] sur les chemins de ce plateau que Ménard avait consacré, pour l'éternité, en paradis terrestre. (Lafon, 2008 : 90-91)

Le jardin étant un symbole du paradis terrestre - «son ami [...] jardinier perpétuel à perte de vue de cet Eden » (91) -, l'analogie est vite établie entre le plateau du Coiron et le Jardin des plantes de Montpellier: «Au fond, le Coiron était un jardin, aussi peu fréquenté que le Jardin des plantes de Montpellier, et non moins inspirateur.» (91) Les promenades en l'honneur de la vie et de l'amitié sont aussi source d'inspiration créatrice vitale, condensée dans le "Cahier de Coiron ». C'est, cette fois, le territoire du paysan ardéchois qui le maintient en vie en tant qu'homme de lettres: "son activité intellectuelle de l'année à venir [...] comme une production naturelle de l'air vivifiant et du ciel imperturbable. » (91) Source d'inspiration possible, ce voyage annuel aux sources de la vie est aussi rêvé et imaginé dans l'attente de son renouvellement rituel.

Le livre de témoignage que Legrand souhaite réaliser sur Ménard le pousse à effectuer ce même voyage pour retrouver les traces du passé et «pour tenter [...] d'ordonner papiers et idées, au cœur de la ferme que dirige aujourd'hui le fils cadet de l'homme qui sauva Ménard de la mitraille (on me reçoit avec la même affection silencieuse que le père manifestait jadis à mon ami) » (93). Par-delà les générations, la vie et la mort, s'ouvre alors un jeu de doubles autour du fils aîné des Vidal, qui se forme à la médicine à Montpellier : «De ce fils et médecin par procuration, de cet héritier et ce double, il tira - de pair avec son ami et sauveteur - certainement une de ses plus grandes fiertés. » (93)

On retrouve la fonction d'amplification du Jardin également dans les motifs narratifs qui lui sont associés : les rencontres, qui finissent toujours par devenir littéraires, le rêve et la rêverie l'amitié, la mort et le double.

\section{L'amitié au fil de la vie}

« (Ne pas en déduire que Ménard voyagea jusqu'au Nouveau Monde, sinon en pensées, en lectures et, pour tout dire, en amitié.) » (Lafon, 2008: 31). Presqu'à toutes les pages, parfois plusieurs fois dans la même page, apparaissent les mots ami(s), amical(e), amitié et leurs dérivés.

7 L'amitié et la mort, la mort et l'amitié sont présentes dans la genèse de la fiction de Borges «Pierre Ménard, auteur du Quichotte». Dans Une vie de Pierre Ménard elles sont aussi intrinsèquement liées à Ménard et au témoignage qu'offre Legrand. De son vivant, les amitiés de Ménard sont littéraires essentiellement. Mais nous venons d'insister sur l'importance du fragment "Rêve d'Ardèche » où apparaît son ami paysan ardéchois et l'amitié fraternelle qui lie les deux hommes : le sauveur et le sauvé. Lorsqu'elle est pensée par rapport à l'absence, l'amitié se fait nostalgie. Les fragments de textes, quel que soit le genre auquel ils appartiennent, rendent l'absent présent: la mort justifie le livre de témoignage ; l'amitié le rend possible.

L'amitié, c'est aussi un Post-scriptum biffé de trois traits et précédé de l'indication «à ne pas lire » écrite à l'encre marron. 


\section{BIBLIOGRAPHIE}

BAKHTINE Mikhail (1978), Esthétique et théorie du roman, Paris : Gallimard.

BORGES Jorge Luis (2013), Ficciones (1 ${ }^{\text {re }}$ éd. 1944), Barcelone : Debolsillo.

LAFON Michel (2008), Une vie de Pierre Ménard, Paris : Gallimard.

LAFON Michel (2009), « Contra el dogma individual », en ligne sur le site de La Nación : <http://

www.lanacion.com.ar/1134851-contra-el-dogma-individual> (05/07/2015).

MANZI Joaquín (2012), «Pensar la tradición después de Borges », en ligne sur le site Academia : < http://www.academia.edu/11851909/Pensar_la_tradici\%C3\%B3n_despu\%C3\%A9s_de_Borges>

MICHAUD François (2011), « La renaissance du Jardin des plantes de Montpellier. Passé, présent et avenir du plus ancien jardin botanique de France ", In Situ, $17 \mid 2011,<10.4000 /$ in situ.3851>, en ligne sur <http://insitu.revues.org/3851> (12 mars 2012).

\section{RÉSUMÉS}

Publié en 2008 aux éditions Gallimard, ce roman-jardin de Michel Lafon s'attache à reconstruire le chemin de vie de l'homme Pierre Ménard, le personnage de la fiction de Borges "Pierre Ménard, auteur du Quichotte ». Le Jardin Botanique de Montpellier, le plus ancien de France, est au cœur de la trame littéraire qui se déploie en fragments de textes pluriels, imprégnés de l'univers littéraire et affectif borgésien. Imprégnés d'amitié aussi. Ce sentiment constitue un véritable fil conducteur de l'œuvre de Jorge Luis Borges à celle de Michel Lafon; de la littérature à la vie et vice-versa ; de la vie à la mort réversible par la grâce et la magie de la fiction.

In this novel-cum-garden, published in 2008 by the Editions Gallimard, Michel Lafon sets out to reconstruct what could have been the life of Pierre Ménard, the protagonist of Borges' "Pierre Menard, Author of The Quixote." The Botanical Gardens of Montpellier - the oldest ones in France - play a pivotal role in a narrative that is composed of fragments of manifold texts, in the true spirit of the literary and emotional atmosphere that is so typical of Borges. Friendship is also given pride of place in this reconstruction, evoking the bonds of friendship that link the works of Jorge Luis Borges and Michel Lafon, from literature to life and vice versa, from life to death, the power of which can be countered through grace and the magic of fiction.

\section{INDEX}

Mots-clés : fiction, roman, Pierre Ménard, jardin, amitié

Keywords : fiction, novel, Pierre Ménard, garden, friendship 
AUTEUR

CARLA FERNANDES

Université Bordeaux Montaigne 